\title{
Demographics, Perceptions, and Use of Medical Marijuana among Patients in Florida
}

\author{
Martha S. Rosenthal ${ }^{a}$ R. Nathan Pipitone ${ }^{b}$ \\ aDepartment of Biological Sciences, Florida Gulf Coast University, Fort Myers, FL, USA; \\ ${ }^{b}$ Department of Psychology, Florida Gulf Coast University, Fort Myers, FL, USA
}

\section{Keywords}

Florida · Medical marijuana · Survey · Demographics ·

Opioids · Education

\begin{abstract}
Medical marijuana (MMJ) is currently legal in 35 US states, with an estimated 3.6 million state-legal medical cannabis patients. Although there are currently over 440,000 MMJ patients in the state of Florida, there are limited data on their demographics, reasons and patterns of use, and successes or failures of treatment. The goal of this study is to examine the demographics, perceptions, and use of medical cannabis among patients in Florida, as well as their perceptions of the quality of information received from physicians and dispensaries. One hundred and fifty-seven MMJ patients completed an online survey regarding their characteristics and use. Patients in Florida shared many characteristics with patients in other states, such as race, use frequency, and administration technique. Patients most commonly used MMJ to treat their symptoms of anxiety, pain, and stress and reported great therapeutic effectiveness. A majority (65\%) of patients also reported either a reduction or total discontinuation of at least one prescription or over-the-counter drug. The find-
\end{abstract}

\begin{tabular}{ll}
\hline karger@karger.com & (c) 2020 The Author(s) \\
Pww.karger.com/mca & $\begin{array}{l}\text { Published by S. Karger AG, Basel } \\
\text { This is an Open Access article licensed under the Creative Commons }\end{array}$ \\
Karger & $\begin{array}{l}\text { Attribution-NonCommercial-4.0 International License (CC BY-NC) } \\
\text { (http://www.karger.com/Services/OpenAccessLicense), applicable to } \\
\text { the online version of the article only. Usage and distribution for com- } \\
\text { mercial purposes requires written permission. }\end{array}$
\end{tabular}

ings highlight the need to further investigate the use of MMJ as a viable treatment for pain and anxiety and to improve access of quality cannabis information to patients, physicians, and dispensary employees.

(C) 2020 The Author(s)

Published by S. Karger AG, Basel

\section{Introduction}

Humans have used cannabis medicinally for at least 5,000 years [1]. Popular for centuries in Asia and Africa, cannabis was considered one of the most important of all medicinal plants. Throughout the 19th and early 20th centuries in Europe and the USA, cannabis was legal and widely available and was used for its therapeutic properties to alleviate pain, stimulate appetite, and promote muscle relaxation. Some political and economic changes in the early 20th century gave rise to swiftly changing attitudes and laws regarding cannabis [1].

In 1970, the Nixon Administration established the Controlled Substances Act, which categorizes controlled substances into 1 of 5 schedules, with schedule I drugs being considered the highest potential for abuse, a lack of accepted safety even under medical supervision, and no 
accepted medical use. Cannabis was categorized as schedule I drug in 1970 and remains there today despite clinical and anecdotal evidence of its safety and therapeutic benefits.

Despite cannabis' controversial status as a schedule I drug, numerous scientific reports have been published regarding cannabis' therapeutic potential. The National Academies of Sciences, Engineering, and Medicine report [2] analyzed over 10,000 studies and concluded that cannabis and/or cannabinoids are effective for treating a variety of symptoms including chronic pain, nausea and vomiting, and stimulating hunger. Today, individuals use medical marijuana (MMJ) to treat a wide range of health conditions, including chronic pain, muscle spasms, anxiety, depression, PTSD, insomnia, headache, nausea, appetite issues, cancer, and the adverse effects of cancer chemotherapy [1,3-6].

MMJ is currently legal in 35 US states, and as of mid2020, there are an estimated 3.6 million state-legal patients [7]. Interest in MMJ is high, and research into North American patient demographics, use, and therapeutic effectiveness of MMJ is increasing. Surveys of MMJ patients have been conducted in California $[6,8,9,12]$, Arizona [8], Michigan [4], and Canada [5]. These studies gathered information on MMJ user demographics, use practices, and level of symptom relief, among other variables.

Findings from the abovementioned work suggest that the typical MMJ patient is white, male, and approximately 35-45 years old; the underrepresentation of minorities is apparent in many of these surveys $[5,6,8]$. Patients typically medicate daily and prefer the inhalational route of administration $[5,6,8,9]$. The most common conditions that participants reported treating with MMJ include pain, back problems, muscle spasms, anxiety, depression, and insomnia $[4-6,8,10]$. Patients also report experiencing either a lot or almost complete relief for many of these symptoms [8]. Previous work has also investigated patient changes in other drug use alongside their MMJ regiment, with a majority of these patients reporting a decrease and/ or complete cessation of some prescription/over-thecounter (OTC) drugs, alcohol, tobacco/nicotine, and/or other illicit substances $[4,5,9,10,12-15]$.

\section{Medical Marijuana in Florida}

With the passage of the Compassionate Medical Cannabis Act in 2014, Florida became the 22nd state to legalize access to MMJ. This act allowed patients suffering from cancer, chronic seizures, or persistent muscle spasms to have access to cannabis products with less than $0.8 \%$ THC and at least $10 \%$ CBD. In 2016, $71 \%$ of Florida voters approved Amendment 2 (Florida Medical Marijuana Legalization Initiative), which expanded access to full-strength medical cannabis to qualified patients, and protected patients, physicians, caregivers, and medical marijuana dispensaries and their staff from criminal prosecutions or civil sanctions under Florida law. Florida is second only to California for the number of MMJ patients, and almost $1.5 \%$ of its population is MMJ patients [11]. Florida's qualifying medical conditions are "cancer, epilepsy, glaucoma, positive status for human immunodeficiency virus, acquired immune deficiency syndrome, posttraumatic stress disorder (PTSD), amyotrophic lateral sclerosis, Crohn's disease, Parkinson's disease, multiple sclerosis, or other debilitating medical conditions of the same kind or class as or comparable to those enumerated and for which a physician believes that the medical use of marijuana would likely outweigh the potential health risks for a patient." This last phrase bears significance, as it gives a physician leeway to approve a patient if he or she believes the patient has a condition comparable to those listed and if the doctor believes marijuana may benefit the patient. For example, this might allow a physician to approve a patient with anxiety, as they may consider this similar to PTSD.

Researchers are only beginning to gather information on Florida patient demographics, patterns of use, and successes or failures of treatment. Reblin and colleagues [12] investigated MMJ use practices among Florida MMJ glioma patients. Brown et al. [3] analyzed the data of adults aged 50 and older who were early adopters of MMJ in Florida and found many were using MMJ to treat musculoskeletal disorders and spasms (48.4\%), chronic pain (45.4\%), cancer (15.5\%), and psychological disorders (including PTSD) (23.2\%). Almost 73\% reported at least some improvement of their condition since initiating treatment.

As the popularity of the "Florida Man" meme suggests, Florida has a unique population. Just 70 years ago, Florida was the second least populated state in the union; today, it is the 3rd most populous. People come to Florida from many different backgrounds and cultures, which gives the state a diverse mix of liberal and conservative, underprivileged, and extremely affluent. In addition, Florida has the greatest percentage of residents aged 65 and older. Because of the unique population and number of patients on the registry, it is imperative to establish a foundation of descriptive information to determine the
Rosenthal/Pipitone 
Fig. 1. Race and ethnicity of MMJ patients compared to Florida population. MMJ, medical marijuana.

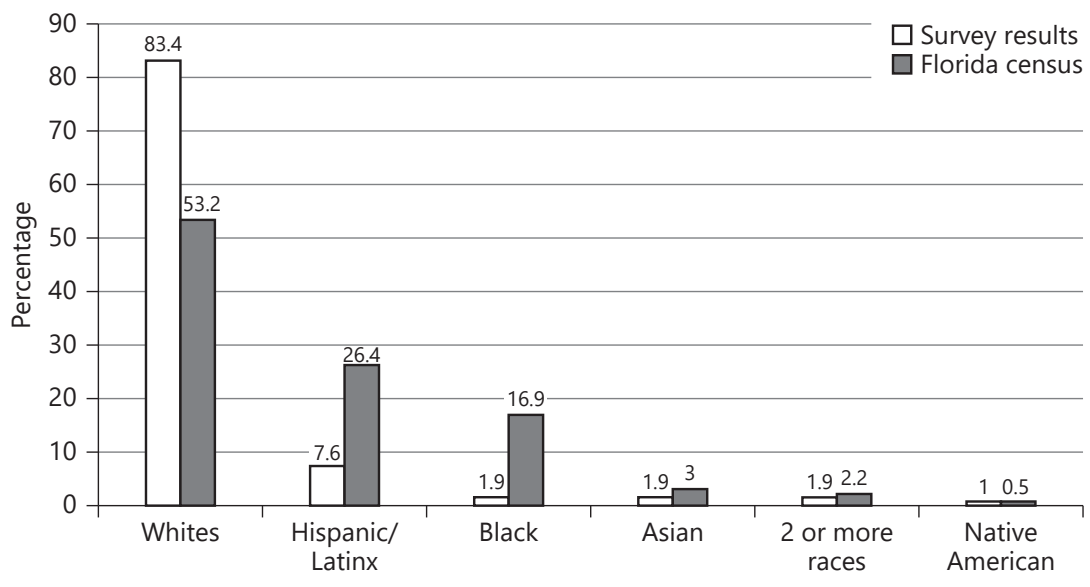

typical use and best practices for MMJ patients in Florida. This paper presents the results of a recent survey so that we may better understand the efficacy of MMJ and the needs of MMJ patients.

\section{Materials and Methods}

The study was cleared by the Florida Gulf Coast University (FGCU) Institutional Review Board (protocol \#2019-19). The online survey tool Checkbox ${ }^{\circledR}$ (https://www.checkbox.com/) was used to collect voluntary survey responses from MMJ patients. Survey solicitation was conducted through Florida's state Medical Marijuana Treatment Centers (MMTC), local Southwest Florida community events, and on FGCU campus. Participants needed to be at least 18 years of age and be a legally registered Florida medical marijuana patient in order to complete the survey. Survey data were collected from May 2019 to January 2020. All subjects gave their informed consent before participation. Upon completion of data collection, the data were cleaned to only include complete responses. Data were analyzed using SPSS ${ }^{\circledR}$ version 26.0 (IBM).

\section{Results}

\section{Patient Demographics}

One hundred and fifty-seven participants (64 male, 93 female) ranging from 21 to 76 years in age $(\mathrm{M}=46, \mathrm{SD}=$ 13.9, with 3 not reporting) completed the survey and were used in analysis. For occupational status, 71 (45.2\%) patients indicated working full time, $48(30.6 \%)$ patients indicated being disabled, $32(20.4 \%)$ patients indicated being retired, $15(9.6 \%)$ patients indicated working parttime, $14(8.9 \%)$ patients indicated being a student, and 7 $(4.5 \%)$ patients indicated being unemployed (note: pa- tients could report $>1$ occupational category at a time; 24 [15.3\%] indicating being in 2 or more occupational categories).

\section{Race and Ethnicity}

In our patient sample, whites were overrepresented and minorities were underrepresented (see Fig. 1). Three individuals preferred not to provide race information.

\section{Use Practices}

Almost all patients surveyed reported using medical cannabis every day. The vast majority of participants $(147 ; 93.6 \%)$ did not feel they have a dependency or addiction to cannabis. Eleven patients (7\%) reported that they needed much less or a little less cannabis than when they started, 41 (26.1\%) patients reported needing about the same now as when they started, $35(22.3 \%)$ patients reported needing a little more or much more than when they started, and $2(1.3 \%)$ patients reported other. Many patients $(68,43.3 \%)$ reported that their use depends on their symptoms. Most participants - 75.2\% - said that they had used marijuana before receiving their MMJ card.

\section{Cannabinoid Ratio}

Patients were asked about the cannabinoid ratios in the strains they used most commonly. Patients could report $>1$ category. The majority of patients indicated that the strains they most commonly use have high THC rather than high CBD: 64 (40.8\%) patients indicated that their strains had high THC levels only, 115 (73.2\%) patients said their strains had high THC/low CBD levels, 56 (35.7\%) reported that their strains had low THC/high 
Table 1. Patient symptoms and perceived symptom relief with MMJ use



MMJ, medical marijuana. * Many patients reported having multiple symptoms.

CBD levels, and 36 (22.9\%) participants said their strains were CBD only, with 4 (2.5\%) reporting not knowing what types of cannabinoids were in their MMJ strains.

\section{Dispensaries}

The participants reported that the dispensaries they purchase most often from are Trulieve (42.3\%), Surterra (18.6\%), and Curaleaf (9.6\%), which are the top dispensaries in the state [11]. Their most popular reasons for purchasing from the dispensaries they visit most frequently include selection of products $(65.6 \%)$, location (47.8\%), convenience $(29.3 \%)$, price $(28.7 \%)$, and staff helpfulness (28\%).

\section{Cost}

Participants reported spending an average of USD 323.6 on MMJ products at dispensaries over the course of 1 month (median $=$ USD 300, SD = USD 240.7). Fortyseven patients did not respond. Two participants reported spending over USD 1,200 per month on medical cannabis. When these outliers were removed, 110 patients reported spending an average of USD 304.9 on MMJ (median $=$ USD 300, SD = USD 197.3).

\section{Routes of Administration}

When asked which route of administration they currently use, participants reported using the following: vaporizer/oil: 128 (81.5\%), smoked flower: 109 (69.4\%), tincture drops: 89 (56.7\%), edibles: 74 (47.1\%), vaporizer/ dry flower: 47 (29.9\%), lotion: $54(34.4 \%)$, capsule: 38 (24.2\%), patch: $31(19.7 \%)$, soft gel: $9(5.7 \%)$, tablet: 7 (4.5\%), and others: 9 (5.7\%).

\section{Patient Symptoms, Perceived Relief, and Side Effects Symptoms}

The top symptoms our survey participants reported treating with MMJ include anxiety, pain, stress, insomnia, and depression (Table 1).

\section{Perceived Relief}

Patients were asked about the extent of relief that MMJ had on their symptoms on a Likert scale from 1 to 5 , with 1 corresponding to no relief at all and 5 corresponding to complete relief. Average symptom relief scores ranged from 4.3 (nausea) to 3.7 (GI distress). Across all symptom relief categories, the percentage of patients who indicated either a good amount of relief (scores of 4 on 1 to 5 scale) or complete relief (scores of 5 on 1 to 5 scale) of their symptoms ranged from $70 \%$ (general inflammation) to $91 \%$ (nausea), with an average percentage of good amount/complete relief being $81.3 \%(\mathrm{SD}=7.4 \%)$. All perceived relief results can be found along with patient symptom percentages in Table 1.

\section{Side Effects}

Patients were asked to check any possible side effects of their MMJ use from a list provided. Participants reported both positive and negative side effects (Fig. 2), al-
16

Med Cannabis Cannabinoids 2021;4:13-20 DOI: $10.1159 / 000512342$
Rosenthal/Pipitone 


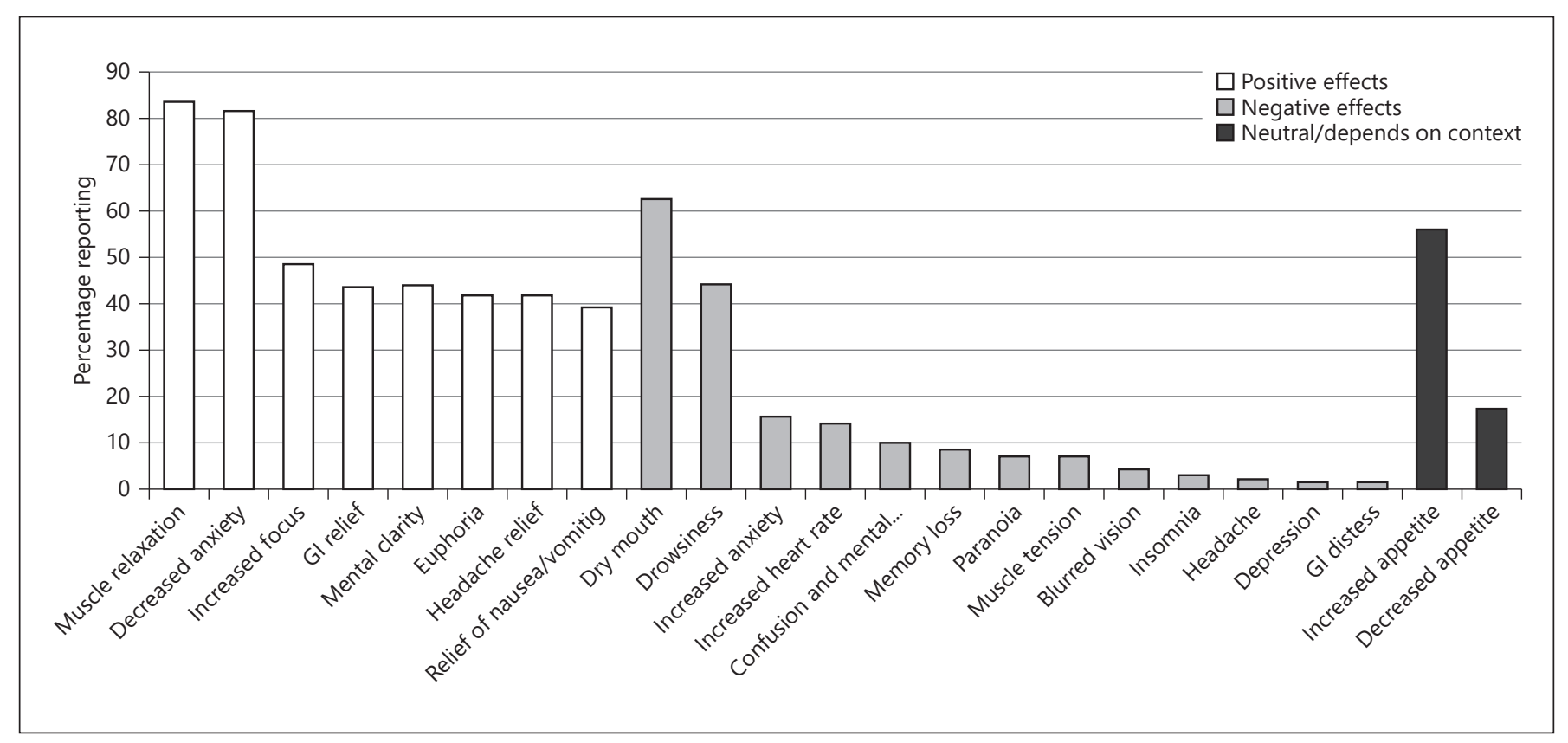

Fig. 2. Side effects reported from MMJ patients in Florida. MMJ, medical marijuana.

though patients were more likely to report positive than negative side effects.

\section{Reduction or Discontinuation of Other Medications}

When asked "since you started using MMJ, has your use (i.e., the amount or frequency) of prescription or OTC medications changed?" $78.3 \%$ of patients reported changes in their prescription or OTC drug use. Unfortunately, $17 \%$ of these patients provided either incomplete information or did not provide the direction of change in their other drug use; therefore, their drug use changes could not be categorized. After eliminating these from the analysis, 102 (65\%) of 157 patients reported either a reduction or total discontinuation of at least one prescription or OTC drug. Forty-one patients (26\% of all surveyed) reported reducing intake of at least one drug, 72 patients ( $46 \%$ of all surveyed) reported discontinuing intake of at least one drug, and 11 patients (7\%) reported both a reduction and discontinuation of a drug (Table 2).

\section{Perception of Guidance Received}

When patients were asked whether they had received adequate information about MMJ from their recommending physician, $75 \%$ of the participants answered in the affirmative. Slightly more, $79.2 \%$, felt they had received adequate guidance from the dispensary. Race (white vs. nonwhite individuals) did impact adequate lev-
Table 2. Percentage of MMJ patients reporting reduced and/or discontinued use of the top 7 prescription and OTC drug categories

\begin{tabular}{lll}
\hline Drug category & $\begin{array}{l}\text { Reduced } \\
\text { use, \% }\end{array}$ & $\begin{array}{l}\text { Dis- } \\
\text { continued } \\
\text { use, \% }\end{array}$ \\
\hline Narcotics/opioids & 20 & 18 \\
Anxiolytics/GABA agonists (antianxiety) & 20 & 18 \\
Antidepressants (e.g., SSRI and SNRI) & 7.3 & 15 \\
Muscle relaxants & 7.3 & 8.6 \\
NSAIDs and nonopioid analgesics & 28 & 7.9 \\
Anticonvulsant & 1.8 & 6.5 \\
Sleep aid & 3.6 & 3.6 \\
\hline
\end{tabular}

The values represent the percentage of patients who indicated reducing drug intake and/or discontinuing drug use in each category. Forty-one patients (26\%) indicated reducing drug intake and 72 patients (46\%) indicated discontinuing at least one drug. Some patients reported reducing and discontinuing drugs in multiple categories. MMJ, medical marijuana; OTC, over-the-counter.

els of information received from physicians, $\chi^{2}(1, n=$ $149)=5.0, p=0.025$. Specifically, $96 \%$ of nonwhite individuals indicated that they received adequate information, compared to $75 \%$ of white individuals. Race did not impact levels of adequate information received from dispensaries, and sex did not impact levels of adequate information received from physicians or dispensaries. 
When asked about specific aspects of MMJ, $45.2 \%$ of patients felt they received adequate guidance from their physician regarding different strains, $50.3 \%$ about the dose, and $47.8 \%$ about routes of administration. Race and sex did not impact this type of information provided by physicians.

\section{Discussion}

With this survey, we establish basic demographic information on patients who use MMJ in the state of Florida and show emerging evidence for the efficacy of MMJ to provide relief on a variety of symptoms. Patient MMJ cannabinoid profiles, use frequency, and location of purchase are also established, as well as their perceptions of information and guidance provided by physicians and dispensaries.

Although previous surveys of cannabis use have found males to be more highly represented among MMJ patients $[4-6,8,9], 59.2 \%$ of our participants were identified as female. Many studies report that women are more likely to seek medical attention and men are more likely to use cannabis recreationally, so it may be that women are more likely to seek cannabis from a medical source while males are more likely to acquire it from a nonmedical source [13]. Or it may simply be the case that women were more likely to take this survey.

The results of this and other work $[6,8]$ show not only that the average MMJ patient is white but also that minority populations are greatly underrepresented by these surveys and perhaps are less likely to use MMJ. One reason could be due to our convenient sampling methodology. Another reason may be fear. Cannabis is still a schedule I drug, and African Americans are 2-4 times more likely to be arrested for marijuana possession than are whites [14, 15]. Given the disproportionate number of people of color in jail for nonviolent drug offensives, perhaps African American and Hispanic/Latinx individuals are also less likely to respond to official surveys regarding current drug use.

Most participants - 75.2\% - said that they had used marijuana before receiving their MMJ card. Other surveys found a similar result $[4,5,8]$. Unlike opioids, where medical use via prescription often precedes recreational use and dependence, the order is typically reversed with MMJ, as patients begin using medical cannabis after they have already experienced recreational use [5]. This makes it difficult to tease out the differences between recreational and medical use of cannabis. It may be that some recreational users are finding symptomatic relief and are self-medicating, but getting their cannabis from nonmedical sources.
Similar to results from other surveys $[5,6,8-10]$, our participants prefer inhalation (smoking and vaping) followed by tinctures and edibles as their route of administration. This may be due to some advantages of the inhalation route of administration. Inhalation allows for faster onset, better control and titration of the dose, and more consistent results than with edibles. The preference for vaping oil over smoking or vaping whole flower may be a factor of availability; flower was not available at Florida dispensaries until 2019, and supplies have been known to run short. Some patients may also prefer the consistency of vape cartridges.

The results also suggest that we may also be underserving those with limited income. The median amount of money patients in the survey spent on MMJ was approximately USD 3,600 per year. The median pretax income in a Florida household is USD 53,267; MMJ expenses would account for $6.8 \%$ of this value. This may explain why some choose to get their product on the (less expensive) illegal market and others may choose to stick with prescription medications, which may not be as effective or which may have distressing side effects, but are covered by insurance.

One particularly important finding from the current survey was that the majority (65\%) of patients reported either a reduction or complete discontinuation of prescription opioid and/or OTC drugs. This complements other work, which has similarly found a reduction in the use of opioids, nonopioid analgesics, benzodiazepines, and antidepressants among MMJ patients [4, 8, 16-19]. These reductions have tremendous implications, as up to 116 million Americans suffer from chronic pain, and physicians often prescribe opioids for their patients [20]. Opioid-related overdose deaths are one of the leading causes of preventable death in the USA, killing about 130 Americans every day [21]. Cannabinoids can inhibit pain through multiple mechanisms and can act synergistically with other pain relievers such as opioids and NSAIDs [1, 22, 23]. Thus, cannabinoids seem to be a safer option to treat chronic pain in some patients. Studies that analyze data from Medicare Part D found that once a MMJ law was implemented in a state, there was a reduction in the use of prescription drugs for which cannabis could serve as a clinical alternative, such as opioids for pain and benzodiazepines for anxiety $[24,25]$. This is significant, as the economic burden of prescription opioid abuse is estimated to be over USD 78 billion [26]. It also seems particularly noteworthy when one considers that prescription drugs typically cost less than MMJ. This discrepancy is especially notable with Medicare, which covers all or almost all of the cost of drugs (e.g., oxycodone, alprazolam, and fluoxetine).
18

Med Cannabis Cannabinoids 2021;4:13-20 DOI: $10.1159 / 000512342$
Rosenthal/Pipitone 
Kruger and Kruger [4] show that MMJ patients in Michigan reported higher levels of trust in using MMJ than mainstream healthcare and report better effectiveness, fewer side effects, and safety outcomes with MMJ than with pharmaceutical substances. These findings might explain why many patients choose to pay out of pocket for medical cannabis rather than using prescriptions drugs, which cost a fraction as much as MMJ.

Among our patients surveyed, $82 \%$ indicated exhibiting symptoms of anxiety and $73 \%$, stress. Of those patients, $87 \%$ and $90 \%$ indicated either a good amount or complete relief of anxiety and stress, respectively. Approximately one-third of Americans will suffer from a significant anxiety disorder at some point in their lives [27]. In 2019, over 44 million Americans - 16\% of the population of those aged 12 and older - reported using tranquilizers or sedatives in the past year and 5.9 million reported misusing them [13]. The number of deaths in the USA attributable to benzodiazepine overdose has risen 10-fold in the past 2 decades; in 2017, benzodiazepines were involved in over $16 \%$ of all fatal drug overdoses in the USA $[28,29]$. Thus, the effectiveness of MMJ in alleviating symptoms of stress and anxiety in our study should be noted.

Considering all patient symptoms, the majority of patients reported having very effective symptom relief from their MMJ use (Table 1), which mirrors results from other patient surveys $[4-6,8,10]$. Specifically, the percentage of patients that indicated either a good amount and/or complete relief of symptoms ranged from $70 \%$ (general inflammation) to $91 \%$ (nausea), with an average percentage of this metric being $81.3 \%$ across all categories. This complements other work, for example, Trout and DiDonato [8] who found that MMJ patients experienced a lot of relief/ almost complete relief for many conditions, including alcohol dependency, anxiety, ADHD, chronic pain, depression, headaches, insomnia, muscle spasms, nausea, and stress. Although patients reported a variety of side effects from their MMJ use, the majority were positive outcomes (e.g., muscle relaxation and decreased anxiety) compared to negative ones (e.g., dry mouth and drowsiness). Moreover, only $4.5 \%$ of patients reported needing much more MMJ over time to maintain its therapeutic effectiveness (17.8\% reported needing a little more) and the vast majority of patients reported having no cannabis dependency or addiction (although subjective measures of substance dependence might be prone to bias). Compared to mainstream pharmaceuticals, which report lower levels of therapeutic effectiveness, more serious side effects, and higher tolerance and addiction, MMJ has potential medicinal benefits.

Survey of Medical Marijuana Patients in Florida
While many patients felt they received adequate information from their physicians, a significant minority did not, and slightly more participants reported receiving adequate information from their dispensary than from their physician. This may be because many physicians do not feel they have the knowledge necessary to accurately inform their patients. In Florida, to become qualified to recommend cannabis to their patients, physicians must go through a 2-h training session - but this session demonstrates how to properly enter the data into the database and does not cover the pharmacokinetics, pharmacodynamics, or physiological effects of cannabis. Furthermore, only 9\% of medical schools in the USA have content on medical cannabis [30]. A survey of medical residents and fellows found that $89.5 \%$ felt "not at all prepared" to prescribe MMJ and 35.3\% felt "not at all prepared" to answer patients' questions about MMJ. Only $7.4 \%$ felt very or extremely prepared to answer questions about MMJ. One review of existing surveys showed that most medical practitioners consider cannabis to be a potential therapeutic option, but rated their self-perceived knowledge about the pharmacology of cannabis to be very low [31]. As medical cannabis becomes increasingly available and utilized, the need for current, accurate, and accessible information about cannabis and its effects becomes more necessary.

The results of this study should be considered in light of some limitations. Patients were largely recruited through medical cannabis dispensaries, cannabis education courses and programs, and cannabis community events. This may lead to a positive bias, as those with more positive outcomes and experiences with $\mathrm{MMJ}$ may be more likely to complete the survey. Although our data recruitment time frame was over the course of 6 months, we ended up with a smaller sample size as compared to other work in this area. Also, our sampling regiment was primarily centered around the Southwest Florida region; therefore, our data might not be completely generalizable to the larger Florida MMJ patient population. Therefore, these data should be considered preliminary in nature. Patients also gave self-reports as to their symptoms and perceptions of relief and were not directly evaluated by a medical professional. Thus, the possibility of placebo effects should not be discounted. However, our survey was anonymous and voluntary in an attempt to limit any systematic bias. When asked whether they received "adequate information" from physicians or dispensaries, patients' responses were subjective. This information still has value, as every patient requires a different level of assistance, and cannabis physicians and dispensary workers should be cognizant of ensuring that all patients feel respected and receive the information they need.

Med Cannabis Cannabinoids 2021;4:13-20 19 
In summary, this work establishes basic demographic information on Florida MMJ patients. Our data support other findings on how MMJ can provide relief from a variety of negative physiological and psychological symptoms and the effectiveness of MMJ to lessen the use of other prescription/OTC drugs.

\section{Statement of Ethics}

All subjects have given their informed consent by providing survey responses. The study protocol was approved by the FGCU Institutional Review Board (protocol \#2019-19).

\section{Conflict of Interest Statement}

The authors have no conflicts of interest to declare.

\section{Funding Sources}

There were no sources of funding for this study.

\section{Author Contributions}

M.S.R. and R.N.P. conceived and designed the survey and IRB protocol. M.S.R. collected the data. R.N.P. analyzed the data. M.S.R. and R.N.P. wrote the manuscript.

\section{References}

1 Rosenthal MS. Drugs: mind, body and society. New York: Oxford University Press; 2018.

2 National Academies of Sciences, Engineering, and Medicine. The health effects of cannabis and cannabinoids. Washington, DC: National Academies Press; 2017.

3 Brown JD, Costales B, van Boemmel-Wegmann S, Goodin AJ, Segal R, Winterstein AG. Characteristics of older adults who were early adopters of medical cannabis in the Florida Medical Marijuana Use Registry. J Clin Med. 2020;9(4):1166

4 Kruger DJ, Kruger JS. Medical cannabis users' comparisons between medical cannabis and mainstream medicine. J Psychoactive Drugs. 2019 Jan-Mar;51(1):31-6.

5 Lucas P, Walsh Z. Medical cannabis access, use, and substitution for prescription opioids and other substances: a survey of authorized medical cannabis patients. Int J Drug Policy. 2017;42:30-5.

6 Reinarman C, Nunberg H, Lanthier F, Heddleston T. Who are medical marijuana patients? Population characteristics from nine California assessment clinics. J Psychoactive Drugs. 2011;43(2):128-35.

7 Marijuana Policy Project. Medical marijuana patient numbers [Internet]. 2020 May 20.

8 Troutt WD, DiDonato MD. Medical cannabis in Arizona: patient characteristics, perceptions, and impressions of medical cannabis legalization. J Psychoactive Drugs. 2015; 47(4):259-66.

9 O'Connell TJ, Bou-Matar CB. Long term marijuana users seeking medical cannabis in California (2001-2007): demographics, social characteristics, patterns of cannabis and other drug use of 4,117 applicants. Harm Reduct J. 2007;4:16-22.

10 Reiman A, Welty M, Solomon P. Cannabis as a substitute for opioid-based pain medication: patient self report. Cannabis Cannabinoid Res. 2017;2(1):160-66.

11 Office of Medical Marijuana Use. OMMU weekly updates [Internet]. 2020 November 27 [updated 2020 November 27, cited 2020 November 27]. Available from: https://knowthefactsmmj.com/about/weekly-updates/.

12 Reblin M, Sahebjam S, Peeri NC, Martinez YC, Thompson Z, Egan KM. Medical cannabis use in glioma patients treated at a comprehensive cancer center in Florida. J Palliat Med. 2019;22(10):1202-7.

13 Substance Abuse and Mental Health Services Administration (SAMHSA). Results from the 2019 national survey on drug use and health: detailed tables [Internet]. Rockville, MD: Center for Behavioral Health Statistics and Quality; 2020.

14 American Civil Liberties Union. The war on marijuana in black and white. New York: ACLU; 2013.

15 The Sentencing Project. Trends in U.S. Corrections [Internet]. 2019 June [updated 2019 June cited 2020 June 25]. Available from: https:// sentencingproject.org/wp-content/uploads/2016/01/Trends-in-US-Corrections.pdf.

16 Reiman A. Medical cannabis patients: patient profiles and health care utilization patterns. Complement Health Pract Rev. 2007;12(1): 31-50.

17 Boehnke KF, Litinas E, Clauw DJ. Medical cannabis use is associated with decreased opiate medication use in a retrospective crosssectional survey of patients with chronic pain. J Pain. 2016;17(6):739-44.

18 Corroon JM, Mischley LK, Sexton M. Cannabis as a substitute for prescription drugs: a cross-sectional study. J Pain Res. 2017;10: 989-98.

19 Vigil JM, Stith SS, Adams IN, Reeve AP. Association between medical cannabis prescription opioid use chronic pain patients: a preliminary report. PLoS One. 2017;12:e0187795.

20 Flexon JL, Stolzenberg L, D’Alessio SJ. The effect of cannabis laws on opioid use. Int J Drug Policy. 2019;74:152-9.

21 Centers for Disease Control and Prevention. Understanding the epidemic [Internet]. 2020 March 19 [updated 2020 March 19 cited 2020
June 24]. Available from: https://www.cdc. gov/drugoverdose/epidemic/index.html.

22 Elikotil J, Gupta P, Gupta K. The analgesic potential of cannabinoids. J Opioid Manag. 2009;5(6):341-57.

23 Hill KP, Palastro MD, Johnson B, Ditre JW. Cannabis and pain: a clinical review. Cannabis Cannabinoid Res. 2017;2(1):96-104.

24 Bradford AC, Bradford WD. Medical marijuana laws reduce prescription medication use in medicare part D. Health Aff. 2016; 35(7):1230-6.

25 Bradford AC, Bradford WD, Abraham A, Adams GB. Association between U.S. state medical cannabis laws and opioid prescribing in the Medicare part D population. JAMA Int Med. 2018;178(5):667-72.

26 Florence CS, Zhou C, Luo F, Xu L. The economic burden of prescription opioid overdose, abuse, and dependence in the United States, 2013. Med Care. 2016;54(10):901-6.

27 Kessler RC, Petukhova M, Sampson NA, Zaslavsky AM, Wittchen H-U. Twelvemonth and lifetime prevalence and lifetime morbid risk of anxiety and mood disorders in the United States. Int J Methods Psychiatr Res. 2012;21(3):169-84.

28 Lembke A, Papac J, Humphreys K. Our other prescription drug problem. N Engl J Med. 2018;378(8):693.

29 National Institute of Drug Abuse. Overdose death rates [Internet]. 2020 March 10 [updated 2020 March 10 cited 2020 June 23]. Available from: https://www.drugabuse.gov/related-topics/trends-statistics/overdose-deathrates.

30 Evanoff AB, Quan T, Dufault C, Awad M, Bierut LJ. Physicians-in-training are not prepared to prescribe medical marijuana. Drug Alcohol Depend. 2017;180:151-5.

31 Gardiner KM, Singleton JA, Sheridan J, Kyle GJ, Nissen LM. Health professional beliefs, knowledge, and concerns surrounding medicinal cannabis: a systematic review. PLoS One. 2019;14(5):e0216556. 